\title{
Ansiedad e insatisfacción corporal en universitarios
}

\author{
MARÍA BEGOÑA MEDINA-GÓMEZ* \\ Universidad de Burgos, España \\ ORCID: https://orcid.org/0000-0002-4772-7798 \\ MARÍA ÁNGELES MARTÍNEZ-MARTÍN \\ Universidad de Burgos, España \\ ORCID: https://orcid.org/0000-0001-6295-7340 \\ MARÍA CAMINO ESCOLAR-LLAMAZARES \\ Universidad de Burgos, España \\ ORCID: https://orcid.org/0000-0003-2448-0267 \\ YOLANDA GONZÁLEZ-ALONSO \\ Universidad de Burgos, España \\ ORCID: https://orcid.org/0000-0003-0818-1996 \\ ELVIRA MERCADO-VAL \\ Universidad de Burgos, España \\ ORCID: https://orcid.org/0000-0001-6142-0154
}

\begin{abstract}
How to quote this article: Medina-Gómez, M.B., Martínez-Martín, M.A., Escolar-Llamazares, M.C., González-Alonso, Y. \& Mercado-Val, E. (2019). Anxiety and body dissatisfaction in university students. Acta Colombiana de Psicología, 22(1), 13-21. doi: http://www.dx.doi.org/10.14718/ ACP.2019.22.1.2
\end{abstract}

Resumen

La ansiedad y la insatisfacción corporal son factores de riesgo para los trastornos de conducta alimentaria y de la ingesta de alimentos. La presente investigación busca analizar los niveles de ansiedad e insatisfacción corporal, y su relación con las diferencias en función del género, curso y centro de estudios en 516 universitarios de Burgos, España -el $42.8 \%$ fueron varones y el $57.2 \%$, mujeres; $M=21.7$ años $(D T=4.1)$-, utilizando un muestreo aleatorio estratificado. Para ello, se realizó un estudio descriptivo transversal en el que se aplicó el Cuestionario de Ansiedad Estado-Rasgo y la subescala Insatisfacción Corporal del Inventario de Trastornos de Conducta Alimentaria. Como resultados, alrededor del $20 \%$ de los participantes presentó niveles altos de ansiedad, aunque no se encontraron diferencias por género, curso o centro; adicionalmente, el $61.4 \%$ presentó alta o moderada insatisfacción corporal, principalmente si eran mujeres, personas ansiosas, o de segundo curso en la universidad. También se encontró interrelación entre estos factores, por lo cual en algunos universitarios se pudo identificar determinado riesgo para la aparición de trastornos de la conducta alimentaria y de la ingesta de alimentos.

Palabras clave: ansiedad, insatisfacción corporal, universitarios, género.

\section{Anxiety and body dissatisfaction in university students}

\begin{abstract}
Anxiety and body dissatisfaction are risk factors for eating behavior disorders (ACT). This research analyzes the levels of anxiety and body dissatisfaction, the relationship between these variables, and the differences according to gender, course and center in 516 university students of Burgos (Spain), using a stratified random sampling. $42.8 \%$ were males and 57.2 $\%$ females, $\mathrm{M}=21.7$ years $(\mathrm{SD}=4.1)$. Was carried descriptive study a cross-sectional. The State-Trait Anxiety Inventory and the subscale Body Dissatisfaction of the Eating Disorders Inventory are applied. About $20 \%$ of participants have high levels of anxiety, not finding differences by gender, course and center. $61.4 \%$ present high and median body dissatisfaction, mainly women, the more anxious and those of 2 nd. In addition, there is an interrelation between factors. Therefore, in some students there are conditioning factors that are risk factors for the appearance of eating behavior disorders.

Keywords: anxiety, body dissatisfaction, university, gender.
\end{abstract}

\footnotetext{
Departamento de Ciencias de la Educación, Facultad de Educación, Universidad de Burgos, C/Villadiego n. ${ }^{\circ}$ 1. 09001, Burgos, España, +34947111422,bmedina@ubu.es
} 


\title{
Ansiedade e insatisfaçáo corporal em universitários
}

\begin{abstract}
Resumo
A ansiedade e a insatisfação corporal são fatores de risco dos transtornos de comportamento alimentar e da ingestão de alimentos. Esta pesquisa procura analisar os níveis de ansiedade e de insatisfação corporal, bem como sua relação com as diferenças em função do gênero, do curso e do centro de estudos em 516 universitários de Burgos, Espanha — 42.8 \% homens e $57.2 \%$, mulheres; $\mathrm{M}=21.7$ anos $(\mathrm{DP}=4.1)$ - utilizando uma amostra aleatória estratificada. Para isso, foi realizado um estudo descritivo transversal, no qual foi aplicado o Inventário de Ansiedade Traço-Estado e a subescala Insatisfação Corporal do Inventário de Transtornos de Comportamento Alimentar. Como resultados, ao redor de $20 \%$ dos participantes apresentaram níveis altos de ansiedade, embora não se tenham encontrado diferenças por gênero, curso ou centro de estudos; além disso, $61.4 \%$ apresentaram alta ou moderada insatisfação corporal, principalmente mulheres, pessoas ansiosas ou de segundo curso na universidade. Também foi encontrada inter-relação entre esses fatores, razão pela qual, em alguns universitários, pôde ser verificado determinado risco para o surgimento de transtorno de comportamento alimentar e da ingestão de alimentos.

Palavras-chave: ansiedade, insatisfação corporal, universitários, gênero.
\end{abstract}

\section{Introducción}

Los estudiantes que se incorporan a la universidad tienen que adaptarse a nuevas rutinas, cambios psicosociales y demandas académicas y sociales de cara a su preparación profesional, todo en un mundo universitario que presenta características y exigencias organizativas y académicas que en ocasiones conducen a cierto nivel de ansiedad (Angelucci, Cañoto \& Hernández, 2017; Emond et al., 2016; Martínez \& Bilbao, 2015).

La ansiedad es un mecanismo adaptativo natural que puede ayudar a afrontar los retos de la vida; sin embargo, cuando se presenta de manera intensa, desproporcionada, continua y de forma en que interfiere con las actividades diarias, se considera desadaptativa (Riveros, Hernández \& Rivera, 2007; Serrano-Pintado \& Escolar-Llamazares, 2015). De acuerdo con Lazarus y Folkman (1986), la ansiedad se define como un estado emocional desagradable que surge como consecuencia de una valoración cognitiva que el sujeto realiza sobre la situación amenazante; es decir, de un análisis de las condiciones ambientales y los recursos personales disponibles para afrontar dicha situación. Y algunos autores, como Spielberger, Gorsuch y Lushene (1982), diferencian entre la Ansiedad-Estado (A-E), que recoge los sentimientos subjetivos de tensión y aprensión que pueden variar con el tiempo y fluctuar en intensidad, y la Ansiedad-Rasgo (A-R), que señala la tendencia relativamente estable de la persona a percibir las situaciones como amenazantes.

En los últimos años, el interés por analizar la ansiedad y sus efectos en el colectivo de estudiantes universitarios ha aumentado considerablemente, tal como se pone de manifiesto en algunas investigaciones centradas en este tema
(Agudelo-Vélez, Casadiegos-Garzón \& Sánchez-Ortiz, 2008; Bayram \& Bilgel, 2008; Doumit et al., 2017). En dichas investigaciones se ha señalado una tasa de ansiedad desadaptativa en universitarios que oscila entre 23-25\% para los hombres y entre $36-42 \%$ para las mujeres, que es de dos a tres veces más alta $(8 \%$ y $15 \%$ respectivamente) si se compara con la población en general (Andrews, Hejdenberg \& Wilding, 2006); mientras que, en cuanto a la edad, los resultados de los estudios no son concluyentes, ya que algunos muestran que los universitarios más jóvenes tienen más ansiedad que los mayores -debido a la dificultad de adaptarse a un nuevo entorno-(Bayram \& Bilgel, 2008), y en otros estudios, principalmente en uno realizado en el Reino Unido -en el que participaron 16460 estudiantes y en el que se trazó el curso longitudinal de la ansiedad a lo largo de la carrera universitaria-, en el cual se reveló que las puntuaciones de ansiedad estudiantil alcanzaron su máximo en el primer y último año, además de que en ningún momento de todo el paso por la universidad descendió el estrés psicológico a los niveles previos a la admisión en la misma (Bewick, Koutsopoulou, Miles, Slaa \& Barkham, 2010).

Por otra parte, se sabe que la ansiedad patológica es una de las emociones más comúnmente asociadas a los trastornos alimentarios y de la ingestión de alimentos (TCA) (Escolar-Llamazares, 2015; Gitimu et al., 2016; Pineda-García, Gómez-Peresmitré, Velasco-Ariza, PlatasAcevedo \& Áramburo-Vizcarra, 2014); pero, aunque no se conoce bien su papel en estos trastornos, sí es una realidad que las personas con trastornos de la alimentación muestran puntuaciones más elevadas en ansiedad que aquellas sin patología alimentaria. En este marco, la mayoría de los trabajos que analizan las relaciones causa-efecto entre 
ansiedad y patología alimentaria consideran la ansiedad como un factor de riesgo para la aparición de dichos trastornos (Solano-Pinto \& Cano-Vindel, 2012); y diferentes estudios que han analizado la incidencia de ansiedad en los TCA señalan comorbilidades que van del 33 al $48 \%$ (Doumit et al., 2017).

Por otro lado, es ampliamente reconocido el impacto que la insatisfacción con la imagen corporal tiene sobre los TCA en jóvenes y su relación con el género (Baños \& Miragall, 2015; Doumit et al., 2017; Lim \& You, 2017; Trujano, De Gracia, Nava \& Limón, 2014). Por ejemplo, García, Solbes, Expósito y Navarro (2012) ponen de manifiesto en un estudio con universitarios españoles que, aunque las mujeres están más insatisfechas con su cuerpo que los hombres, a estos también les preocupa y esa preocupación surge en todas las edades. En este sentido, Lameiras, Calado, Rodríguez y Fernández (2003) constataron que el $38.6 \%$ de mujeres y el $19.1 \%$ de varones realizaron conductas de control del peso para mejorar su imagen corporal al menos una vez en los últimos seis meses. Para estos mismos autores, si bien no se puede considerar que el descontento con la imagen corporal sea, por sí solo, causante de un TCA, sí es un factor de riesgo responsable del inicio y mantenimiento de las conductas extremas para controlar el peso. De igual forma, se ha encontrado que estas conductas pueden conducir al desarrollo de un TCA en personas vulnerables, y que constituye uno de los factores que con más consistencia y robustez contribuyen al diagnóstico, inicio y desarrollo de la patología alimentaria en universitarios (Berengüí, Castejón \& Torregrosa, 2016; García et al., 2012).

En particular, la insatisfacción corporal se caracteriza por la presencia de juicios valorativos sobre el cuerpo que no coinciden con las características reales (Berengüi et al., 2016), y varios estudios la han asociado con la ansiedad (Cruz, Pascual, Salaberria \& Echeburua, 2015; Cruz, Pascual, Wlodarczyk, Polo \& Echeburúa, 2016; Doumit et al., 2017; Koronczai et al., 2013). Por ejemplo, Cruz et al., (2016) indican que cuanto mayor es la insatisfacción corporal, mayor es el nivel de ansiedad; y que, por tanto, estar satisfecho con el propio cuerpo podría considerarse como un factor protector de sintomatología ansiosa (Dooley, Fitzgerald \& Giollabhui, 2015). Asimismo, la insatisfacción corporal también se ha relacionado con distorsiones en la alimentación cuando el nivel de ansiedad es alto (Doumit et al., 2017); y, en resumen, estas aportaciones sugieren la existencia de un vínculo entre la insatisfacción con la imagen corporal y la ansiedad, de manera que tanto una imagen corporal deficiente puede contribuir a mayor ansiedad, como, a su vez, la ansiedad puede conducir a un TCA (Doumit et al., 2017).
Sin embargo, escasas investigaciones han analizado la relación entre ansiedad e insatisfacción corporal en función del curso y la carrera, y estas no son concluyentes (EscolarLlamazares et al., 2017). Por mencionar algunas, Guedea, Ornelas, Rodríguez y Gastétum (2012) revelan que los estudiantes de educación física manifiestan mayores niveles de ansiedad en relación con su cuerpo que los alumnos de ciencias de la salud; mientras que Aguiar, Rodrigues y de Pinho (2014) indican que la incidencia de factores de riesgo para presentar un TCA es mayor en universitarios de grados pertenecientes al área de salud (como Nutrición y Dietética, Educación Física, Enfermería y Medicina), donde la apariencia física y la preocupación por el bienestar son muy importantes.

Ahora bien, dada la importante relación entre ansiedad e insatisfacción con la imagen corporal como factores de riesgo para los TCA en población universitaria, el presente estudio se centra en analizar: (1) si los estudiantes de la Universidad de Burgos (España) tienen altos niveles de ansiedad e insatisfacción con su propio cuerpo; (2) si existe alguna relación entre las variables A-E, A-R y la insatisfacción corporal; y (3) si la ansiedad e insatisfacción con la imagen de estos estudiantes difiere en función del género, curso y centro de estudios.

\section{Método}

\section{Tipo de estudio}

Se diseñó un estudio descriptivo transversal por medio de una encuesta con una muestra probabilística (Montero \& León, 2007).

\section{Participantes}

Se llevó a cabo un muestreo aleatorio estratificado entre los 26 grados impartidos en los siete centros de la Universidad de Burgos, con una población de 6277 alumnos. La muestra estuvo constituida por 516 participantes, y este cálculo se realizó utilizando la fórmula destinada a muestras finitas estratificadas, con un nivel de confianza del $95.6 \%$ y un margen de error del $4 \%$. En resumen, el $42.8 \%$ de la muestra eran varones $(n=240)$ y el $57.2 \%$, mujeres $(n=321)$, ambos entre los 17 y los 65 años $(M=21.7, D T=4.1)$; además, el $75 \%$ de los universitarios procedía de Burgos y provincia, el $12 \%$ del resto de provincias españolas y el $13 \%$ de otros países (China, México y Francia); y el $37.1 \%$ de estaba matriculado en cuarto curso, el $28.5 \%$ en segundo, el $21.7 \%$ en tercero y el $12.7 \%$ en primero. En la Tabla 1 se presentan las frecuencias y porcentajes de estudiantes por curso y por centro de estudios. 
Tabla 1.

Frecuencia y porcentaje de alumnos por curso y centro de estudios

\begin{tabular}{llllllll}
\hline \multirow{2}{*}{ Curso } & $\begin{array}{l}\text { Ciencias } \\
\mathrm{n}(\%)\end{array}$ & $\begin{array}{l}\text { DRL } \\
\mathrm{n}(\%)\end{array}$ & $\begin{array}{l}\text { Económicas } \\
\mathrm{n}(\%)\end{array}$ & $\begin{array}{l}\text { Educación } \\
\mathrm{n}(\%)\end{array}$ & $\begin{array}{l}\text { EPS } \\
\mathrm{n}(\%)\end{array}$ & $\begin{array}{l}\text { HCA } \\
\mathrm{n}(\%)\end{array}$ & $\begin{array}{l}\text { Salud } \\
\mathrm{n}(\%)\end{array}$ \\
\hline 1 & - & $6(7.1)$ & $1(1.5)$ & $28(23.3)$ & $5(3.4)$ & $9(17.0)$ & $22(46.8)$ \\
2 & $14(33.3)$ & - & $3(44.8)$ & $49(40.8)$ & $57(38.5)$ & $9(17.0)$ & $1(2.1)$ \\
3 & $28(66.7)$ & $7(8.33)$ & $22(32.8)$ & $6(15)$ & $27(18.2)$ & $8(15.1)$ & $24(51.1)$ \\
4 & - & $71(84.5)$ & $14(20.9)$ & - & $59(39.9)$ & $27(50.9)$ & - \\
\hline
\end{tabular}

Nota . DRL = Derecho y Relaciones Laborales; EPS = Escuela Politécnica Superior; HUCA = Humanidades y Comunicación Audiovisual.

\section{Instrumentos}

Se elaboró un cuestionario ad hoc denominado "Cuestionario de Información sobre Conducta Alimentaria, Ansiedad y Depresión", conformado por dos partes: una destinada a obtener datos sociodemográficos (género, edad, lugar de residencia, centro, grado y curso), y otra formada por dos instrumentos estandarizados: el Inventario de Ansiedad Estado-Rasgo (STAI E/R) de Spielberger et al. (1982) y el Inventario de Trastornos de la Conducta Alimentaria-Cuestionario de Remisión (EDI-3-RF), de Garner (2004). En este trabajo se presentan los resultados obtenidos con el análisis del STAI E/R y de la subescala de Insatisfacción Corporal (BD) del EDI-3-RF.

Inventario de Ansiedad Estado-Rasgo.

El State-Trait Anxiety Inventory (STAI), de Spielberger et al. (1982), en su adaptación de Seisdedos (1994), se trata de un autoinforme de 40 ítems que evalúan dos tipos de ansiedad: la Ansiedad-Estado (A-E; condición emocional transitoria) y la Ansiedad-Rasgo (A-R; tendencia ansiosa relativamente estable). Las dos subescalas están constituidas por 20 ítems en un sistema de calificación tipo Likert de cuatro puntos (donde $0=$ nada, $1=$ algo, 2 = bastante, $y$ 3 = mucho), diseñadas para ser administradas individual o colectivamente. En muestras de población española, el cuestionario tiene altos índices de consistencia interna (entre .90 y .93 en A-E y entre .84 y .87 en A-R) y correlaciones altas con la Escala de Ansiedad de Cattell (entre .73 y .85). En el manual de la prueba no se indica a partir de qué percentil se considera una ansiedad alta, por lo que se siguió el criterio de Martínez-Otero (2014) que considera el umbral de ansiedad alta a partir de percentiles superiores a 75 .

Subescala de Insatisfacción Corporal (BD) del Cuestionario de Remisión del Inventario de Trastornos de la Conducta Alimentaria.

El Eating Disorder Inventory (EDI-3-RF), de Garner (2004), en su adaptación española de Elosua, López y
Sánchez (2010), es una subescala conformada por 10 ítems autoaplicados que evalúan la insatisfacción con la forma general del cuerpo y con el tamaño de partes concretas del mismo (estómago, caderas, muslos y nalgas, por ejemplo), en un rango de puntuación directa que va de 0 a 40 . Además, esta subescala cuenta con un rango cualitativo de insatisfacción personal (de 0-6, baja insatisfacción, de 7-27, media, y de 28-40, alta). En cuanto a los datos psicométricos, se obtuvieron valores entre .82 y $.96 \mathrm{de}$ consistencia interna (alfa de Cronbach) en la adaptación española para muestras clínicas españolas, y entre .64 y .92 para muestras no clínicas. En la muestra del presente estudio, el valor de consistencia interna obtenido para la subescala de Insatisfacción Corporal (BD) fue de .84 .

\section{Procedimiento}

Se aplicaron las pruebas en los distintos centros educativos durante los meses de noviembre y diciembre de 2015 , tras contactar previamente a los docentes de los grupos seleccionados aleatoriamente. El día asignado para administrar las pruebas, un miembro del equipo investigador, acompañado por el docente del grupo, facilitó el cuadernillo con los instrumentos a cada uno de los participantes. La duración aproximada de la aplicación de las pruebas fue de 15-30 minutos.

\section{Consideraciones éticas}

Antes de dar respuesta a las preguntas del cuadernillo, uno de los investigadores informó a los participantes sobre los objetivos y la confidencialidad del estudio, indicó que la colaboración era voluntaria, solicitó el consentimiento informado y permaneció en el aula con el fin de resolver las dudas que pudieran surgir.

\section{Análisis de datos}

Una vez aplicadas las pruebas, y tras seguir las normas de corrección de los cuestionarios, se obtuvo la puntuación directa y los percentiles en el STAI, y se prosiguió a 
la caracterización de las puntuaciones del BD en los tres rangos cualitativos. Los datos se codificaron y analizaron con el programa estadístico SPSS v.22.

En primer lugar, se analizó el porcentaje de alumnos que obtuvo altos niveles en las pruebas aplicadas, $\mathrm{y}$, posteriormente, se realizó el cálculo de correlaciones (Pearson) entre las puntuaciones de las subescalas contempladas y, después, el estudio de la regresión lineal mediante el método de pasos sucesivos. Se empleó un intervalo de confianza del $95 \%$ y un nivel de significación de $p<.05$.

Antes de realizar el estudio de las diferencias en función del género, curso y centro de estudio, se comprobó con la prueba de Kolmogorov-Smirnov que las variables no se ajustaron a una distribución normal en $\mathrm{A}-\mathrm{E}(Z=.12 ; p=.00)$, en A-R $(Z=.09 ; p=.00)$, ni en $\operatorname{BD}(Z=.10 ; p=.00)$. Posteriormente, se comprobó que los índices de simetría $(\mathrm{A}-\mathrm{E}=-.01, \mathrm{~A}-\mathrm{R}=-.29$ y $\mathrm{BD}=-1.34)$ y curtosis $(\mathrm{A}-\mathrm{E}=$ $1.41, \mathrm{~A}-\mathrm{R}=.91$ y $\mathrm{BD}=.43)$ tienden a estar próximos a la normalidad (valores < 2) (Curran, West \& Finch, 1996). En los casos en que no se asumió la igualdad de varianzas (Prueba de Levene), se emplearon pruebas no paramétricas: U de Mann Whitney en las variables con dos niveles de comparación (género) y Kruskal Wallis en las variables con más de dos alternativas de respuesta (curso y centro). Cuando se asumió la igualdad de varianzas, la prueba utilizada fue la $t$ de Student (género). Todas las pruebas contaron con un nivel de significancia de .05 .

\section{Resultados}

A continuación se presenta la descripción de los resultados a partir de los estadísticos de los valores de las diferentes escalas del STAI E/R y de la subescala de Insatisfacción Corporal (BD) del EDI-3-RF.

En lo relacionado con el primer objetivo de este estudio -analizar los niveles de ansiedad e insatisfacción corporal del alumnado universitario-, en la Tabla 2 se recogen la frecuencia y el porcentaje de alumnos de la muestra total en cada uno de los percentiles de las dos escalas del STAI y en los rangos establecidos en la subescala de insatisfacción corporal (BD).

Con respecto a la ansiedad, el $18.4 \%$ de la muestra obtuvo puntuaciones por encima del perfil 75 en A-E, mientras el $21 \%$ lo obtuvo en A-R; y en la insatisfacción corporal, el $61.4 \%$ presentó valores altos y moderados, frente al $38.6 \%$ de los universitarios que manifestó estar satisfecho con su cuerpo.

En cuanto al segundo objetivo-analizar si existe relación entre las variables A-E, A-R y la insatisfacción corporal-, se obtuvo una correlación significativa entre los dos tipos
Tabla 2.

Frecuencia y porcentaje en las subescalas de ansiedad y en la insatisfacción corporal

\begin{tabular}{|c|c|c|c|c|}
\hline \multicolumn{5}{|c|}{ Ansiedad } \\
\hline \multirow{2}{*}{ Percentiles } & \multicolumn{2}{|c|}{ A-E } & \multicolumn{2}{|c|}{ A-R } \\
\hline & $\mathrm{n}$ & $\%$ & $\mathrm{n}$ & $\%$ \\
\hline$<25$ & 190 & 33.8 & 160 & 28.5 \\
\hline $26-50$ & 119 & 21.2 & 136 & 24.2 \\
\hline $51-75$ & 150 & 26.7 & 148 & 26.3 \\
\hline $76-90$ & 83 & 14.8 & 89 & 15.8 \\
\hline$>91$ & 20 & 3.6 & 29 & 5.2 \\
\hline \multicolumn{5}{|c|}{ Insatisfacción corporal (BD) } \\
\hline \multicolumn{3}{|c|}{ Rango } & $\mathrm{n}$ & $\%$ \\
\hline \multicolumn{3}{|c|}{ Baja (0-6) } & 217 & 38.6 \\
\hline \multicolumn{3}{|c|}{ Moderada (7-27) } & 329 & 58.5 \\
\hline \multicolumn{3}{|c|}{ Alta $(28-40)$} & 16 & 2.9 \\
\hline
\end{tabular}

Nota . A-E $=$ Subescala de Ansiedad-Estado; A-R = Subescala de Ansiedad-Rasgo.

de ansiedad $(r=.704 ; p<.000)$, en donde, al hacer el análisis de regresión lineal, el $49.5 \%$ de la varianza de la A-E es explicada por la A-R $(B=.698, \beta=.704 ; t=23.428$; $p<.001)$; mientras que al analizar la correlación de insatisfacción corporal con las otras dos variables se observa una correlación, aunque no muy elevada, entre la insatisfacción corporal y la A-E $(r=.345 ; p=.000)$, y entre la insatisfacción corporal y la A-R $(r=.386 ; p=.000)$. En el primer caso, si se realiza una regresión lineal, el $12 \%$ de varianza en insatisfacción personal es explicada por A-E $(B=.093 ; \beta=.345: t=8.676 ; p<.001)$ y el $15 \%$ por A-R $(B=.103 ; \beta=.386, t=9.906 ; p<.001)$.

Con respecto al tercer objetivo de esta investigación -analizar si la ansiedad y la insatisfacción con la imagen corporal de estos estudiantes difiere en función del género, curso y centro de estudios-, se encontró que, en cuanto al género, los resultados en la prueba de Levene indicaron puntuaciones de $F=.535$ y $p=.469$ en A-E; $F=2.550$ y $p=.111$ en A-R; y $F=18.532$ y $p=.000$ en BD. Asimismo, se aplicó la prueba U de Mann Whitney y no se encontraron diferencias significativas entre hombres y mujeres con respecto a la ansiedad, pero sí al utilizar la $t$ de Student frente a la insatisfacción corporal (véase Tabla 3). De este modo, se encontró que las mujeres obtuvieron mayores puntuaciones en la subescala $\mathrm{BD}(M=11.61 ; D T=8.112)$ que los varones $(M=7.83 ; D T=6.613)$, $\mathrm{y}$, al calcular el tamaño del efecto con la $d$ de Cohen, se obtuvo un valor de .51, es decir, un efecto moderado (Cohen, 1988).

También, con respecto al curso, se comprobó que no existe homogeneidad de varianzas en ninguna de las 
variables: $\mathrm{A}-\mathrm{E}(F=.816 ; p=.485), \mathrm{A}-\mathrm{R}(F=.546 ; p=.651)$ y $\mathrm{BD}(F=.361 ; p=.781)$, por lo que se aplicó la prueba no paramétrica de Kruskal Wallis, y, como se observa en la Tabla 3, se encontró que existen diferencias de medias en insatisfacción con la imagen corporal únicamente entre los alumnos de segundo y tercer curso $(M=11.61 ; D T=8.109$ y $M=9.00 ; D T=7.293$, respectivamente).

Por último, con respecto al centro donde cursan estudios, una vez comprobada la no homogeneidad de varianzas: A-E $(F=.995 ; p=.428)$, A-R $(F=1.498 ; p=.177)$ y BD $(F=1.299 ; p=.256)$, se aplicó la prueba Kruskall-Wallis a un nivel de significancia del .05 , y se encontró que no existen diferencias significativas en ninguna de las variables (véase Tabla 3).

Tabla 3.

Diferencias en las subescalas de ansiedad e insatisfacción corporal según género, curso y centro

\begin{tabular}{ccccccc}
\hline & $\mathrm{A}-\mathrm{E}$ & \multicolumn{2}{c}{$\mathrm{A}-\mathrm{R}$} & \multicolumn{2}{c}{$\mathrm{BD}$} \\
\hline & $\mathrm{U}$ & $\mathrm{p}$ & $\mathrm{U}$ & $\mathrm{p}$ & $\mathrm{t}$ & $\mathrm{p}$ \\
\hline Género & 36794.500 & .363 & 38512.000 & .997 & 5.910 & .000 \\
\hline & $\chi^{2}$ & $\mathrm{p}$ & $\chi^{2}$ & $\mathrm{p}$ & $\chi^{2}$ & $\mathrm{p}$ \\
\hline Curso & 2.104 & .147 & .426 & .514 & 4.747 & .029 \\
Centro & 3.227 & .072 & .015 & .903 & .220 & .639 \\
\hline
\end{tabular}

Nota. A-E $=$ Subescala de Ansiedad Estado; $\mathrm{A}-\mathrm{R}=$ Subescala de Ansiedad Rasgo; BD = Subescala de Insatisfacción Corporal.

\section{Discusión}

El presente estudio analiza la relación existente entre las variables ansiedad e insatisfacción corporal, considerándolas como factores que aumentan el riesgo de sufrir un TCA. Con este objetivo, el trabajo se centra en examinar la presencia de ambas variables en el colectivo de estudiantes de la Universidad de Burgos; la relación existente entre los tipos de ansiedad (A-E y A-R) y la insatisfacción corporal; y las diferencias en estas variables en función de algunas características sociodemográficas (género, curso, centro).

Los resultados observados indican que existe una proporción importante de universitarios con altos niveles de A-E (18.4\%) y de A-R (21\%); datos similares a los obtenidos en otro estudio con universitarios españoles que recoge porcentajes de 13.20 en A-E y 16 en A-R (Martínez-Otero, 2014). Esto puede deberse, por una parte, a que los universitarios se enfrentan a situaciones con niveles de exigencia valorados emocionalmente como amenazantes y, por otra, a que crean que no tienen los recursos personales necesarios para afrontarlos. También, se debe tener en cuentan que, en general, las personas con alta A-R presentan una A-E más elevada porque tienden a percibir las situaciones cotidianas como más amenazantes o peligrosas (Spielberger et al., 1982).

En cuanto a la variable insatisfacción corporal, un alto porcentaje de estudiantes $(61.4 \%)$ manifestó un nivel moderado y alto de insatisfacción con su cuerpo, lo cual está en concordancia con estudios previos que muestran que más del $60 \%$ de los estudiantes universitarios apuntan hacia una insatisfacción corporal elevada (García et al., 2012; Soto-Ruiz et al., 2015). Los resultados tanto en ansiedad como en insatisfacción con el propio cuerpo indican que un grupo importante de universitarios presentan puntuaciones elevadas en las pruebas aplicadas con el riesgo que esto supone para su salud.

Como era de esperar, se observa una interacción entre los dos tipos de ansiedad (A-E y A-R), de manera que las influencias ambientales del entorno universitario que generan tensión emocional en los universitarios son mayores en aquellos que tienen una personalidad caracterizada por rasgos más ansiosos. Al analizar la relación existente entre la ansiedad y la insatisfacción corporal, se obtiene, aunque esta sea baja, posiblemente debido a la multicolinealidad entre las variables -ya que cuanto mayores son los niveles de ansiedad (A-E y A-R), mayor es la posibilidad de estar insatisfecho con el propio cuerpo-, y más si la ansiedad es una característica de personalidad más o menos estable (A-R). Por lo tanto, aquellos estudiantes más ansiosos podrían estar más insatisfechos con su cuerpo, y esa insatisfacción conllevaría a la realización de hábitos no saludables que puedan desencadenar un TCA. Estos resultados son consecuentes con los obtenidos en una muestra de 765 mujeres españolas entre los 16-20 años, según los cuales, cuanto mayor es el nivel de ansiedad, mayor es la insatisfacción corporal; lo que conlleva el incremento de conductas no saludables para el control del peso (Cruz et al., 2016).

Por otra parte, al estudiar la influencia de características sociodemográficas, en el presente estudio no se confirman los resultados obtenidos por otros autores en los que se encuentra que las mujeres presentan mayores niveles de ansiedad -atribuidos a la influencia de factores fisiológicos, dificultades sociales, la asignación de diversos roles sociales o el estrés causado por el ambiente- (Riveros et al., 2007). Es posible que las estudiantes de la Universidad de Burgos no vivencien el ambiente universitario de forma más amenazante que los varones, y que, por esto, no sea el género la variable influenciable en el nivel de ansiedad; lo cual puede deberse a que las dificultades sociales y las exigencias académicas son similares para los alumnos de ambos sexos, y que, además, los roles sociales asignados a las mujeres a esa edad no difieren sustancialmente a los atribuidos a los varones. En este sentido, se debe considerar 
que el $75 \%$ de la muestra reside en Burgos o sus alrededores y que por esto las rutinas diarias han podido verse poco modificadas.

En cambio, el malestar y descontento con su imagen sí es mayor en las estudiantes mujeres. En este sentido, es probable que la presión social general sobre la apariencia y la insatisfacción con la imagen corporal por no cumplir con los estándares sociales sobrepase la influencia que el ambiente universitario tiene sobre las mujeres. Estos resultados coinciden con los obtenidos por otros autores que afirman que las estudiantes muestran mayor insatisfacción con su físico y perciben su imagen corporal menos positivamente que los varones (Clode, Lewis \& Fuller-Tyszkiewicz, 2016). Estas puntuaciones deben ser especialmente tenidas en cuenta, pues la insatisfacción corporal es uno de los factores más consistentes y robustos que contribuyen al desarrollo de la patología alimentaria en los universitarios (Baños \& Miragall, 2015); y la insatisfacción con su propio cuerpo en compañía de altos niveles de ansiedad puede ocasionar comportamientos poco saludables que supongan consecuencias perjudiciales en la vida cotidiana de las mujeres, como lo es seguir dietas sin supervisión médica, por ejemplo (Olesti-Baiges et al., 2007).

Adicionalmente, con base en los resultados obtenidos en este estudio respecto al curso académico, no aparecen diferencias significativas en la ansiedad; hallazgo que va en contra a lo encontrado por Bayram y Bilgel (2008) con respecto a la ansiedad A-E, puesto que los alumnos de primer curso en su estudio presentaron mayores niveles ansiedad debido a la necesidad de adaptarse a un nuevo entorno. La falta de variabilidad en A-E en el presente estudio puede deberse al momento de aplicación del cuestionario, ya que se produjo entre los meses de noviembre y diciembre, y las exigencias académicas en este periodo lectivo son diferentes a las de épocas de exámenes, ya que se ha encontrado que los estudiantes muestran un aumento en los niveles de ansiedad durante estos períodos (Macht, Haupt \& Eligring, 2005).

En este mismo sentido, Shamsuddin et al. (2013) encontraron niveles de ansiedad mayores en alumnos de último curso por su preocupación hacia el futuro; pero en los universitarios burgaleses del presente estudio no se observó mayor ansiedad en los estudiantes de cuarto curso, probablemente porque cuando se aplicaron los cuestionarios, los estudiantes no estaban vivenciando la inmediatez de la búsqueda de un empleo o planteándose el futuro a medio plazo. Esto podría interpretarse como que las valoraciones de las exigencias académicas son similares en todos los alumnos independientemente del curso en que están matriculados, al menos durante el periodo de docencia. Sería interesante aplicar los instrumentos de evaluación en distintos momentos del curso escolar para analizar si la A-E se ve influenciada por acontecimientos concretos de la vida universitaria (por ejemplo, al inicio del curso, en el periodo de exámenes y a la finalización de la carrera). En este trabajo, la media más alta en insatisfacción corporal correspondió a los alumnos de segundo curso, y la diferencia con estudiantes de otros grupos es significativa únicamente con los de tercero, pero ante este hecho no encontramos justificación.

Por último, respecto a si existen diferencias en ansiedad e insatisfacción corporal en función del centro de estudios, en el presente estudio tampoco se encontraron diferencias en ningún tipo de ansiedad ni en la insatisfacción corporal. En este sentido, no parece que el grado que estudian sea una característica determinante a la hora de hacer valoraciones cognitivas desagradables sobre una situación amenazante, ni subjetivas sobre la imagen corporal. Los resultados del presente estudio no son coincidentes con los de Aguiar et al. (2014), quien afirma que los universitarios de grados que tienen que ver con la salud tienen niveles más altos en la ansiedad como factor de riesgo para la patología alimentaria.

Como conclusión, se puede señalar que a la Universidad de Burgos acuden un número elevado estudiantes que experimentan altos niveles de ansiedad y aún más elevado con moderada o alta insatisfacción corporal; factores que, como se sabe, aumentan el riesgo a sufrir un TCA. Además, la insatisfacción corporal y la ansiedad (en sus dos manifestaciones) correlacionaron significativamente, lo que confirma la presencia de vulnerabilidad dentro de este colectivo -mayor en las mujeres, pues estas presentan mayores niveles de insatisfacción corporal-, tal como se recoge en investigaciones previas (Escolar-Llamazares et al., 2017; Martínez \& Bilbao, 2015). Por lo tanto, en un grupo de universitario se da una serie de condicionantes que pueden desencadenar un problema de salud en el que sería conveniente intervenir lo antes posible.

Esta información resulta de gran interés, ya que facilita el abordaje a nivel preventivo de ambas variables (ansiedad e insatisfacción corporal) de forma conjunta. La identificación temprana de posibles problemas de ansiedad -que en ciertas ocasiones están ligados a moderada y alta insatisfacción corporal- evitaría el desarrollo de otras patologías como las relacionadas con los TCA. Desde una perspectiva de la salud pública, el conocimiento de la prevalencia de problemas de salud mental en los jóvenes universitarios españoles es esencial, por lo que es preciso seguir investigando en el área para obtener más información que permita alertar a la sociedad sobre el riesgo al que están expuestos los estudiantes universitarios y poder plantear programas adecuados de detección e intervención temprana destinados a mejorar su calidad de vida y que permitan aumentar su rendimiento académico, y, sobre todo, su satisfacción personal. 
A pesar de lo anterior, este trabajo no está exento de limitaciones, y una de ella es no haber realizado un seguimiento para evaluar si los resultados obtenidos se mantienen en el tiempo. Asimismo, es importante señalar que, aunque el presente estudio se ha centrado en analizar la relación entre la ansiedad y la insatisfacción corporal, sería interesante estudiar la relación entre ansiedad y otras variables implicadas en la aparición de un TCA, como la obsesión por la delgadez y el comportamiento bulímico, aspectos que serán objeto de otro trabajo. Finalmente, aunque en algunas de las variables analizadas los resultados no son concluyentes, este estudio contribuye a dar visibilidad a posibles problemas de salud mental enmascarados en algunos universitarios.

\section{Referencias}

Agudelo-Vélez, D. M., Casadiegos-Garzón, C. P., \& SánchezOrtíz, D. L. (2008). Características de ansiedad y depresión en estudiantes universitarios. International Journal of Psychological Research, 1(1), 34-39.

Aguiar, J., Rodrigues, C. R., \& De Pinho, L. (2014). Factors associated with the risk of eating disorders among academics in the area of health. Revista Gaúcha de Enfermagem, 35(2), 73-78. doi: https://doi.org/10.1590/19831447.2014.02.42441

Andrews, B., Hejdenberg, J., \& Wilding, J. (2006). Student anxiety and depression: comparison of questionnaire and interview assessments. Journal of Affective Disorders, 95(1), 29-34. doi: http://dx.doi.org/10.1016/j.jad.2006.05.003

Angelucci, L. T., Cañoto, Y., \& Hernández, M. J. (2017). Influencia del estilo de vida, el sexo, la edad y el IMC sobre la salud física y psicológica en jóvenes universitarios. Avances en Psicología Latinoamericana, 35(3), 531-546. doi: http://dx.doi.org/10.12804/10.12804/revistas.urosario. edu.co/apl/a.4454

Baños, R. M., \& Miragall, M. (2015). Los trastornos alimentarios y sus causas. En Autor (Ed.), Todo sobre los Trastornos de la Conducta Alimentaria. Una visión multidisciplinar desde la experiencia y la evidencia cientifica (pp. 49-96). Tarragona: Altaria.

Bayram, N., \& Bilgel, N. (2008). The prevalence and sociodemographic correlations of depression, anxiety and stress among a group of university students. Social Psychiatry and Psychiatric Epidemiology, 43(8), 667-672. doi: 10.1007/ s00127-008-0345-X

Berengüí, R., Castejón, M. Á., \& Torregrosa, M. S. (2016). Body dissatisfaction, risk behaviors and eating disorders in university students. Revista Mexicana de Trastornos Alimentarios, 7(1), 1-8. doi: https://doi.org/10.1016/j. rmta.2016.02.004
Bewick, B., Koutsopoulou, G., Miles, J., Slaa, E., \& Barkham, M. (2010). Changes in undergraduate students' psychological well-being as they progress through university. Studies in Higher Education 35(6), 633-645. doi: http://dx.doi. org/10.1080/03075070903216643

Clode, J., Lewis, V., \& Fuller-Tyszkiewicz, M. (2016). Body Image Concerns as Predictors of Psychology Students' Confidence with Clients. Journal of Applied Biobehavioral Research, 21(4), 253-261.

Cohen, M. A. (1988). Some new evidence on the seriousness of crime. Criminology, 26(2), 343-353. doi: 10.1111/j.17459125.1988.tb00845.x

Cruz, S., Pascual, A., Salaberria, K., \& Echeburúa, E. (2015). Normal-weight and overweight female adolescents with and without extreme weight-control behaviours: Emotional distress and body image concerns. Journal of Health Psychology, 20(6), 730-740. doi: 10.1177/1359105315580214

Cruz, S., Pascual, A., Wlodarczyk, A., Polo-López, R., \& Echeburría, E. (2016). Insatisfacción corporal y conductas de control del peso en chicas adolescentes con sobrepeso: papel mediador de la ansiedad y la depresión. Nutrición Hospitalaria, 33(4), 935-940. doi: http://dx.doi.org/10.20960/ nh.395

Curran, P. J., West, S. G., \& Finch, J. F. (1996). The robustness of test statistics to nonnormality and specification error in confirmatory factor analysis. Psychological Methods, 1(1), 16. doi: http://dx.doi.org/10.1037/1082-989X.1.1.16

Dooley, B., Fitzgerald, A., \& Giollabhui, N. M. (2015). The risk and protective factors associated with depression and anxiety in a national sample of Irish adolescents. Irish Journal of Psychological Medicine, 32(1), 93-105. doi:10.1017/ ipm. 2014.83

Doumit, R., Khazen, G., Katsounari, I., Kazandjian, C., Long, J., \& Zeeni, N. (2017). Investigating vulnerability for developing eating disorders in a multiconfessional population. Community Mental Health Journal, 53(1), 107-116. doi: 10.1007/s10597-015-9872-6

Emond, M., Ten Eycke, K., Kosmerly, S., Robinson, A. L., Stillar, A., \& Van Blyderveen, S. (2016). The effect of academic stress and attachment stress on stress-eaters and stress-undereaters. Appetite, 100, 210-215. doi: https:/doi. org/10.1016/j.appet.2016.01.035

Escolar-Llamazares, M. C. (2015). TCA y su comorbilidad con los trastornos depresivos y de ansiedad. En M. Á. Martínez Martín (Ed.), Todo sobre los Trastornos de la Conducta Alimentaria. Una visión multidisciplinar desde la experiencia y la evidencia cientifica. (pp. 215-232). Madrid: Altaria.

Escolar-Llamazares, M. C., Martínez, M. Á., González, M. Y., Medina, M. B. Mercado, E., \& Lara, F. (2017). Factores de riesgo de trastornos de la conducta alimentaria entre universitarios: Estimación de vulnerabilidad por sexo y edad. Revista Mexicana de Trastornos Alimentarios, 8(2), 105-112. doi: https://doi.org/10.1016/j.rmta.2017.05.003 
García, J., Solbes, I., Expósito, E., \& Navarro, E. (2012). Imagen corporal y riesgo de trastornos de la conducta alimentaria en población universitaria española: diferencias de género. Revista de Orientación Educacional, 26(50), 31-46.

Garner, D. M. (2004). Eating Disorder Inventory-3. Referral Form Manual. Florida, EE. UU.: Psychological Assessment Resources, Inc. (Adaptación española, Elosua, P., López, A., \& Sánchez, F (2010). Inventario de trastornos de la Conducta Alimentaria. Cuestionario de Revisión). Madrid: TEA.

Gitimu, P. N., Jameson, M. M., Turel, T., Pohle-Krauza, R., Mincher, J., Rowlands, Z., \& Elias, J. (2016). Appearance issues, depression, and disordered eating among college females. Cogent Psychology, 3(1), 1-16. doi: http://dx.doi.org /10.1080/23311908.2016.1196512

Guedea, J., Ornelas, M., Rodríguez, J. M., \& Gastétum, G. (2012). Invarianza factorial de la escala de ansiedad asociada a la imagen corporal en estudiantes universitarios de Educación Física y Ciencias de la Salud. Formación Universitaria, 5(6), 39-50. doi: http://dx.doi.org/10.4067/ S0718-50062012000600005

Koronczai, B., Kökönyei, G., Urbán, R., Kun, B., Pápay, O., Nagygyörgy, K., ... Demetrovics, Z. (2013). The mediating effect of self-esteem, depression and anxiety between satisfaction with body appearance and problematic internet use. The American Journal of Drug and Alcohol Abuse, 39(4), 259-265. doi: 10.3109/00952990.2013.803111

Lameiras, M., Calado, M., Rodríguez, Y., \& Fernández, M. (2003). Hábitos alimentarios e imagen corporal en estudiantes universitarios sin trastornos alimentarios. International Journal of Clinical and Health Psychology, 3(1), 23-33.

Lazarus, R. S., \& Folkman, S. (1986). Estrés y procesos cognitivos. Barcelona: Ediciones Martínez Roca, S.A.

Lim, S. A., \& You, S. (2017). Effects of self-esteem and depression on abnormal eating behavior among Korean female college students: mediating role of body dissatisfaction. Journal of Child and Family Studies, 26, 176-182. doi: 10.1007/s10826-016-0542-2

Macht, M., Haupt, C., \& Ellgring, H. (2005). The perceived function of eating is changed during examination stress: a field study. Eating Behaviors, 6(2), 109-112. doi: https:// doi.org/10.1016/j.eatbeh.2004.09.001

Martínez, M. A., \& Bilbao, M. C. (2015). Los trastornos de la conducta alimentaria en el contexto universitario. En M. A. Martínez-Martín (Eds.), Todo sobre los trastornos de la conducta alimentaria: Una visión multidisciplinar desde la experiencia y la evidencia científica (pp. 535-564). Tarragona, España: Altaria.

Martínez-Otero, V. (2014). Ansiedad en estudiantes universitarios: estudio de una muestra de alumnos de la Facultad de Educación. Revista de la Facultad de Educación de Albacete, 29(2), 63-78. doi: http://dx.doi.org/10.18239/ensayos. v29i2.384
Montero, I., \& León, O. G. (2007). A guide for naming research studies in Psychology. International Journal of Clinical and Health Psychology, 7(3), 847-862.

Olesti-Baiges, M., Martín-Vergara, N., Riera-Solé, A., de la Fuente-García, M., Bofarull-Bosch, J. M., Ricomá-de Castellarnau, G., \& Moreso, J. L. P. (2007). Valoración de la propia imagen corporal en adolescentes femeninas de 12 a 21 años de la ciudad de Reus. Enfermería clínica, 17(2), 7884. doi: https://doi.org/10.1016/S1130-8621(07)71773-4

Pineda-García, G., Gómez-Peresmitré, G., Velasco-Ariza, V., Platas-Acevedo, S., \& Áramburo-Vizcarra, V. (2014). Riesgo de anorexia y bulimia en función de la ansiedad y la edad pubertad en universitarios de Baja California-México. Revista Mexicana de Trastornos Alimentarios, 5, 80-90.

Riveros, M., Hernández, H., \& Rivera, J. (2007). Niveles de depresión y ansiedad en estudiantes universitarios de Lima Metropolitana. Revista de Investigación en Psicología, 10(1), 91-102.

Serrano-Pintado, I., \& Escolar-Llamazares, M. C. (2015). Cómo vencer tus problemas de ansiedad ante los exámenes. Madrid: Ediciones Pirámide.

Shamsuddin, K., Fadzil, F., Ismail, W. S. W., Shah, S. A., Omar, K., Muhammad, N. A., ... Mahadevan, R. (2013). Correlates of depression, anxiety and stress among Malaysian university students. Asian Journal of Psychiatry, 6(4), 318323. doi: https://doi.org/10.1016/j.ajp.2013.01.014

Solano-Pinto, N., \& Cano-Vindel, A. (2012). Ansiedad en los trastornos alimentarios: Un estudio comparativo. Psicothema, 24(3), 384-389.

Soto-Ruiz, M. N., Marin-Fernández, B., Aguinaga-Ontoso, I., Guillén-Grima, F., Serrano-Mozó, I., Canga-Armayor, N., ... Annaa, J. (2015). Análisis de la percepción de la imagen corporal que tienen los estudiantes universitarios de Navarra. Nutrición Hospitalaria, 31(5), 2269-2275. doi: 10.3305/nh.2015.31.5.7418.

Spielberger, C. D. Gorsuch, R. I., \& Lushene, R. E. (1970). STAI, Manual for the State-Trait Anxiety Inventory (Self Evaluation Questionnaire). California: Consulting Psychologists press, Inc [Adaptación española de Seisdedos, N. (1994). Cuestionario de ansiedad Estado-Rasgo, STAI. Madrid: TEA].

Spielberger, C. D., Gorsuch, R. L., \& Lushene, R. (1982). Manual del Cuestionario de Ansiedad Estado/Rasgo (STAI). Madrid, España: TEA Ediciones.

Trujano, P., De Gracia, M., Nava, C., \& Limón, G.R. (2014). Sobrepeso y obesidad en preadolescentes mexicanos: estudio descriptivo, variables correlacionadas y directrices de prevención. Acta Colombiana de Psicología, 17(1), 119130. doi: 10.14718/ACP.2014.17.1.12 